\title{
Mortality and cancer incidence in the perfumery and flavour industry of Geneva
}

\author{
E GUBERAN' ${ }^{1}$ AND L RAYMOND ${ }^{2}$ \\ From the Geneva Medical Inspectorate of Factories, ' 1207 Geneva, and the Geneva Cancer Registry, ${ }^{2} 1205$ \\ Geneva, Switzerland
}

\begin{abstract}
An analysis has been made of the mortality and cancer incidence of 1168 workers who entered the three factories of the perfumery industry of the Canton of Geneva from their establishment at the turn of the century to the end of 1964 . The workers were followed up from their entry until 31 December 1980, at which date 344 were dead and 28 lost to follow up. Among the whole study population only mortality from tuberculosis was significantly raised; there was no significant increase in the incidence of or mortality from any cancer. Analysis by four exposure categories showed a significant excess of deaths from heart disease among the compounders. In addition, two deaths from aplastic anaemia were recorded in chemical process workers exposed to benzene. Further analysis by cohort of entry and by birth cohort indicated that, among the subgroup of men first employed in 1900-29 and born in 1880-99, the mortality from a wide range of causes was significantly increased below the age of 70 . This increase is unlikely to be due to an occupational factor as indicated by the absence of an upward trend of mortality with longer exposure.
\end{abstract}

Around the turn of the century three companies processing natural products and manufacturing fine chemicals for perfumery were set up in the Canton of Geneva: Chuit and Naef in 1895 which subsequently took the name Firmenich, Givaudan in 1899 , and "Usines de l'Allondon" in 1917. The last company was absorbed by Firmenich in 1951 . Affiliated companies have been created in various European countries as well as in North and Latin America and in the Far East, to the extent that Givaudan and Firmenich today occupy respectively the second and fourth places worldwide in this branch of the chemical industry.

The production of fine chemicals, and the processing of natural products, requires the use of many solvents and chemical reagents. Process solvents have included benzene, toluene, cyclohexane, methanol, ethanol, diethyl ether, and ethyl acetate. The diversity of chemical reagents used by the industry spans the vast range of organic, organometallic, and inorganic substances that are common to the fine chemical industry in general. These have included some alkylating agents such as ethylene oxide and dimethyl sulphate which are

Received 7 November 1983

Accepted 11 July 1984 suspect carcinogens from short term tests and from animal experiments but for which there is inadequate evidence for carcinogenicity to man, according to the International Agency for Research on Cancer.'

The aroma chemicals that are finally incorporated into perfumes are by necessity relatively inert organic chemicals with molecular weights usually between 100 and 300 and are most commonly aldehydes, ketones, alcohols, ethers, and esters of saturated, unsaturated, or aromatic hydrocarbons.

According to their function, workers came into varying degrees of contact with these substances. The workers whom we have studied may be divided into four groups as follows: (a) chemical process workers who could come into contact with any of about 3000 chemical substances which are used as chemical reagents, solvents, starting materials, intermediates, and finished aroma chemicals, $(b)$ compounders and associated workers who would have their exposure limited to 2000 or so aroma chemicals and essential oils from which are composed the mixtures for incorporation as perfumes into consumer products, $(c)$ maintenance workers who could be exposed on an irregular basis to any of the above chemicals and particularly to cleaning solvents including benzene until the 1960 s, and $(d)$ 
chemical research workers whose range of exposure could extend to over 6000 different substances.

It is also important to mention that most of the aroma chemicals and essential oils to which workers might be exposed have not varied during the present study.

The objective of the study was to examine within the limits of the size of the population concerned, whether there is any evidence of harmful long term occupational effects by comparing the mortality and the cancer incidence of male workers in the three factories with those of the population of Geneva.

\section{Methods}

Complete personnel records since the establishment of the factories were supplied to the Geneva Medical Inspectorate of Factories by the Firmenich and Givaudan companies. The study was based on the 2318 male workers who had entered the Geneva perfumery industry up until 31 December 1964 . The following categories were excluded: 237 clerks, 796 workers employed less than one year, 66 workers born before 1880 , and 51 "frontaliers" * who could not be included because of the difficulty of tracing them in France and the impossibility of obtaining death certificates from that country.

The remaining 1168 men were traced from the time they entered the industry until their death, or the date at which all trace was lost, or 31 December 1980. Among the 1006 Swiss workers, 41 had emigrated and were traced through members of the family remaining in Geneva or Swiss Consulates, or both; only two were lost to follow up. Of the 162 foreign workers, 26 who had left Switzerland were lost to follow up. All the workers were retrospectively classified into four broad exposure categories, except for $52(4 \%)$ for whom the information was insufficient (table 1).

${ }^{*}$ Foreign workers commuting every day into Swiss territory from neighbouring regions of France.

Table 1 Study population. Number of workers by period of entry and by category of exposure

\begin{tabular}{lllll}
\hline Exposure category & \multicolumn{2}{l}{ Period of entry } & \multicolumn{2}{c}{ Total } \\
\cline { 2 - 4 } & $1900-29$ & $1930-49$ & $1950-64$ & \\
\hline Chemical process & 154 & 237 & 197 & 588 \\
Compounding & 14 & 47 & 94 & 155 \\
Maintenance & 53 & 86 & 125 & 264 \\
Research & 17 & 27 & 65 & 109 \\
Unknown & 36 & 14 & 2 & 52 \\
Total & 274 & 411 & 483 & 1168 \\
\hline
\end{tabular}

\section{MORTALITY}

Causes of death were obtained from the death certificates supplied by the Swiss "Office Fédéral de la Statistique." Since 1951 the underlying cause of death has been coded on the certificate according to the International Classification of Diseases (ICD) for the purpose of the annual publication of national mortality statistics. Thirteen workers died in foreign countries, of whom five died while travelling and eight after emigration. For six of these 13 workers the cause of death was obtained from the death certificate and for another three, complete medical histories were supplied by the hospital in which they had died. Detailed information supplied by close relatives was considered sufficiently reliable for the remaining four deaths: one cancer of the larynx, one recurrence of myocardial infarction, and two "sudden deaths."

General mortality was analysed for the period 1900-80 and the cause of death for 1942-80. Before 1942 death certificates were not kept by the Swiss Federal Office of Statistics. Of the total of 344 deaths, 24 occurred before 1942. Person-years at risk were computed with the program called Man Years Computer Language. ${ }^{2}$ Expected numbers of deaths were calculated using annual age specific death rates for Switzerland and adjusted to make allowance for regional differences in mortality, using the ratio Canton of Geneva/Swiss age standardised mortality rates. For the specific causes of death the ratio was averaged for 1969 to 1980 (Geneva cause specific mortality was not available before 1969) and multiplied by the expected number of deaths based on national rates.

\section{CANCER INCIDENCE}

The cases of cancer among the 785 male employees who were resident in the Canton of Geneva were collected by the Geneva Cancer Registry from 1 January 1970 (the date of the start of the registry) to 31 December 1980. Expected numbers of cases of cancer were calculated using Geneva age specific incidence rates, established by the registry, by sex and matrimonial status.

The one sided significance of any excess of observed deaths or cases of cancer was tested assuming a Poisson distribution with a mean equal to the expected value. The normal approximation of the Poisson distribution was assumed where the expected number was greater than 14.0.

\section{Results}

OVERALL MORTALITY

Among the 1168 men (33 938 person-years at risk) there were 344 observed deaths from 1900 to 1980 
compared with an expected of 343.8 . The latter figure does not take into account the first 12 months (or year 0) of follow up. Owing to the criteria of at least one year of exposure for inclusion in the study a man would be precluded if he had died during that year. For the years 1 to 14 since entering the industry the SMR from all causes of death was $76(90 \%$ confidence interval $=59-97$ ) with 46 observed deaths and 60.4 expected deaths, whereas the SMR was $105(95-116)$ for the years 15 and over $(0=$ $298, E=283.4)$. The lower mortality for the first 15 years is probably due to a "healthy population selection effect." 3 To control this bias, subsequent analysis was restricted to the period of follow up starting at the fifteenth anniversary of entry into the industry.
As shown in table 2 no statistically significant excess of observed mortality or incidence for any cancer was found, and the total number of cases of cancer for 1970 to 1980 was lower than expected. Among the non-malignant causes of death (table 3) an excess of observed deaths, significant at the 5\% level, was recorded for tuberculosis. The excess mortality from chronic bronchitis and emphysema, which was not significant $(p=0.07)$, was mainly due to three deaths $(0.7$ expected) among men more than 80 years old, an age where death certification is the least reliable.

MORTALITY BY DURATION OF EXPOSURE

Fox and Collier have pointed out that to obtain an unbiased measure of the effect of exposure duration

Table 2 Cancer mortality 1942-80, and cancer incidence* 1970-80, for men alive 15 years after entry

\begin{tabular}{|c|c|c|c|c|c|c|c|c|}
\hline \multirow[t]{2}{*}{ Site (ICD code, 8th rev) } & \multicolumn{4}{|c|}{ Deaths $1942-80$} & \multicolumn{4}{|c|}{ Cases of cancer $1970-80$} \\
\hline & Obs & $\operatorname{Exp}$ & $S M R$ & $90 \% \mathrm{CI}$ & Obs & $\operatorname{Exp}$ & $S M R$ & $90 \% C I$ \\
\hline All neoplasms (140-239) & 83 & $81 \cdot 7$ & 102 & $84-122$ & 49 & $64 \cdot 1$ & 76 & $59-97$ \\
\hline Buccal cavity, pharynx (140-149) & 6 & $4 \cdot 3$ & 140 & $61-275$ & 4 & 3.8 & 105 & $36-241$ \\
\hline Oesophagus (150) & 7 & $4 \cdot 2$ & 167 & $78-313$ & 2 & $1 \cdot 4$ & 143 & $25-449$ \\
\hline Stomach (151) & 8 & $7 \cdot 4$ & 108 & $54-195$ & 0 & $3 \cdot 8$ & 0 & $0-79$ \\
\hline Intestine, rectum (152-154) & 8 & $9 \cdot 1$ & 88 & 44-159 & 10 & $7 \cdot 8$ & 128 & $70-217$ \\
\hline Liver (155) & 3 & $2 \cdot 9$ & 103 & $28-267$ & 3 & $2 \cdot 1$ & 143 & $39-369$ \\
\hline Gall bladder (156) & 2 & 0.8 & 250 & $43-786$ & 1 & 0.5 & 200 & $8-945$ \\
\hline Pancreas (157) & 6 & $3 \cdot 3$ & 182 & $79-359$ & 2 & $1 \cdot 4$ & 143 & $25-449$ \\
\hline Larynx (161) & 3 & $2 \cdot 5$ & 120 & $32-310$ & 2 & $2 \cdot 0$ & 100 & $17-314$ \\
\hline Lung (162) & 19 & 19.8 & 96 & $63-141$ & 11 & $13 \cdot 4$ & 82 & $46-136$ \\
\hline Skin (172-173) & 0 & $1 \cdot 2$ & 0 & $0-250$ & 5 & $8 \cdot 5$ & 59 & $23-124$ \\
\hline Prostate (185) & 4 & $6 \cdot 7$ & 60 & $20-137$ & 5 & $6 \cdot 7$ & 75 & $29-157$ \\
\hline Bladder (188) & 1 & $3 \cdot 4$ & 29 & $1-139$ & 2 & $3 \cdot 8$ & 53 & $9-165$ \\
\hline Kidney, other urinary organs (189) & 3 & $1 \cdot 9$ & 158 & $43-408$ & $\overline{1}$ & $2 \cdot 0$ & 50 & $2-236$ \\
\hline Brain (191) & 0 & $1 \cdot 7$ & 0 & $0-176$ & $\mathbf{0}$ & 0.8 & 0 & $0-375$ \\
\hline Lymph/haemoporetic (200-209) & 7 & $5 \cdot 3$ & 132 & $62-248$ & $\mathbf{0}$ & $3 \cdot 3$ & 0 & $0-91$ \\
\hline Hodgkin's disease (201) & 2 & 0.9 & 222 & $38-698$ & $\mathbf{0}$ & $0 \cdot 2$ & $\mathbf{0}$ & $0-1498$ \\
\hline Leukaemia (204-207) & 3 & $2 \cdot 0$ & 150 & $41-387$ & 0 & $1 \cdot 1$ & 0 & $0-272$ \\
\hline Others & 6 & $7 \cdot 2$ & 83 & $36-164$ & 1 & $2 \cdot 8$ & 36 & $1-169$ \\
\hline
\end{tabular}

$\mathrm{CI}=$ Confidence interval.

*Except in situ cancers.

Table 3 Mortality 1942-80 from all causes and from non-malignant causes for men alive 15 years after entry

\begin{tabular}{lrrrr}
\hline Cause of death (ICD code, 8th rev) & Obs & Exp & $S M R$ & $90 \%$ CI \\
\hline All causes (0-999) & 286 & $275 \cdot 3$ & 104 & $94-115$ \\
Tuberculosis (010-019) & 10 & $5 \cdot 3$ & $189^{*}$ & $102-320$ \\
Diabetes mellitus (250) & 4 & $3 \cdot 6$ & 11 & $38-254$ \\
Heart disease (390-398, 410-429) & 67 & $66 \cdot 3$ & 101 & $82-124$ \\
Hypertensive disease (400-404) & 3 & $3 \cdot 0$ & 100 & $27-258$ \\
Cerebrovascular disease (430-438) & 20 & $17 \cdot 4$ & 115 & $76-167$ \\
Other circulatory diseases (440-458) & 9 & $8 \cdot 3$ & 108 & $57-189$ \\
Chronic bronchitis, emphysema & 9 & $5 \cdot 1$ & 176 & $92-308$ \\
$\quad$ (491-492) & 5 & $2 \cdot 6$ & 192 & $76-404$ \\
Peptic ulcer (531-533) & 13 & $10 \cdot 3$ & $75-201$ \\
Cirrhosis of liver (571) & 4 & 85 & $29-195$ \\
Motor vehicle accidents (E 810-823) & & 151 & $84-249$ \\
Other accidents and poisonings & $11 \dagger$ & $7 \cdot 3$ & $25-135$ \\
(E 800-807, 825-949) & 5 & $7 \cdot 8$ & 84 & $63-107$ \\
Suicide (E 950-959) & 43 & 51.9 & 83 & \\
Other non-malignant causes & & & \\
\hline
\end{tabular}

$\mathrm{CI}=$ Confidence interval.

* $\mathrm{p}<0.05$.

$\dagger$ Two deaths from aplastic anaemia due to chronic benzene poisoning. 
Table 4 Mortality 1900-80 from all causes in relation to the duration of exposure, for men alive 15 years after entry

\begin{tabular}{lcccr}
\hline Years of exposure & Obs & Exp & SMR & \multicolumn{1}{c}{$90 \%$ CI } \\
\hline $1-4$ & 71 & $66 \cdot 7$ & 106 & $87-130$ \\
$5-9$ & 30 & $20 \cdot 8$ & $144^{*}$ & $104-196$ \\
$10-14$ & 13 & $16 \cdot 6$ & 78 & $46-124$ \\
$\geqslant 15$ & 184 & $179 \cdot 3$ & 103 & $91-116$ \\
\hline
\end{tabular}

$\mathrm{CI}=$ Confidence interval.

${ }^{*} p<0.05$.

the exposure and follow up periods should not overlap. ${ }^{3}$ Therefore the comparison of main interest in table 4 is between exposures of one to four years, five to nine years, and 10 to 14 years among men alive 15 years after entry. There is no evidence of a trend in SMR with longer exposure.

\section{MORTALITY BY CATEGORY OF EXPOSURE}

Analysis by exposure category indicated no significant increase in mortality from the leading categories of death (table 5) or from any specific cause in chemical process workers, maintenance workers, or chemical research workers. Among the process workers, however, the cause of two deaths was "aplastic anaemia due to chronic benzene poisoning." These deaths occurred in 1943 and 1945 at ages of 54 and 57. Medical records obtained from the Geneva University Hospital indicated that both workers were exposed daily for more than 10 years to benzene, had experienced a first episode of aplastic anaemia followed by complete recovery, and had then had a relapse after several years of re-exposure. Necropsies showed generalised symptoms of anaemia, and no other cause of death was found. Medical experts concluded that the causal relationship between exposure to benzene and aplastic anaemia was certain in both cases. Leukaemia mortality, which may be increased by exposure to benzene,' was not excessive among chemical process workers $(0=1, E=1 \cdot 1)$, although the small number of deaths precludes a valid interpretation.

Among the compounders there was a significant excess of deaths from circulatory disease (table 5) which was accounted for mainly by the increased mortality from heart disease $(0=12, E=4 \cdot 8, S M R$ $=250,90 \% \mathrm{CI}=144-405)$. More detailed analysis has to take into account the changes made in the International Classification of Diseases. No death from heart disease was observed for the years 1942-50 $(E=0 \cdot 3)$. Under the sixth and seventh revisions, during the years 1951-68, there were six deaths $(E=1.5)$ from "arteriosclerotic and degenerative heart disease" (ICD 420-422) and three deaths $(E=0.4)$ from "other diseases of the heart" (ICD 430-434). Scrutiny of the death certificates from the first six cases shows that the underlying cause of death was ischaemic heart disease (IHD) in four and "degenerative heart disease" in the remaining two. Between 1969 and 1980 , under the eighth revision, two deaths $(E=1 \cdot 8)$ were due to IHD (ICD 410-414) and one $(E=0.8)$ to "other forms of heart disease" (ICD 420-429). In conclusion the excess of deaths from heart disease occurred mainly in the 1950 s and the 1960 s and was due to IHD as well as to other heart diseases.

Making allowance for a latency of 20 years, the SMR from heart disease was 500 among the compounders exposed one to nine years $(90 \% \mathrm{CI}=$ $20-2360, \mathrm{O}=1, \mathrm{E}=0 \cdot 2), 286(90 \% \mathrm{CI}=49-898$, $\mathrm{O}=2, \mathrm{E}=0.7$ ) among those exposed 10 to 19 years, and $219(90 \% \mathrm{CI}=103-411, \mathrm{O}=7, \mathrm{E}=$ 3.2 ) among those still in the industry 20 years after entry. Thus the excess risk does not increase with longer exposure, although the numbers of deaths are small in the first two exposure categories. (Two of the 12 deaths from heart disease were excluded from this analysis because they occurred between 15 and 20 years since first employment.)

There were in total 101 chemists of whom 36 were associated with chemical processing and eight with compounding whereas 57 were from the chemical research laboratories. Their low mortality from all causes $(\mathrm{O}=29, \mathrm{E}=38 \cdot 6, \mathrm{SMR}=75,90 \% \mathrm{CI}=$ 54-102) is similar to that observed for Swedish and American chemists. ${ }^{45}$ Nevertheless, the significant increase in cancer mortality found among the Swedish chemists was not observed in the present study

Ible 5 Mortality 1942-80 from the leading categories of deaths by exposure category, for men alive 15 years after entry

\begin{tabular}{|c|c|c|c|c|c|c|c|c|c|c|c|c|c|c|c|c|}
\hline \multirow[t]{2}{*}{ use of death } & \multicolumn{4}{|c|}{ Chemical process } & \multicolumn{4}{|c|}{ Compounding } & \multicolumn{4}{|c|}{ Maintenance } & \multicolumn{4}{|c|}{ Research } \\
\hline & Obs & $\operatorname{Exp}$ & $S M R$ & $90 \% C I$ & Obs & $\operatorname{Exp}$ & $S M R$ & $90 \% C I$ & Obs & $\operatorname{Exp}$ & $S M R$ & $90 \% C I$ & Obs & $\operatorname{Exp}$ & $S M R$ & $90 \% \mathrm{CI}$ \\
\hline $\begin{array}{l}\text { I neoplasms } \\
\text { culatory disease } \\
\text { Jlent deaths } \\
\text { her causes } \\
\text { I causes }\end{array}$ & $\begin{array}{r}39 \\
54 \\
13 \\
46 \\
152\end{array}$ & $\begin{array}{r}45 \cdot 7 \\
53 \cdot 7 \\
11 \cdot 8 \\
41 \cdot 3 \\
152.5\end{array}$ & $\begin{array}{r}85 \\
101 \\
110 \\
111 \\
100\end{array}$ & $\begin{array}{l}64-111 \\
79-126 \\
65-175 \\
86-142 \\
87-114\end{array}$ & $\begin{array}{r}6 \\
16 \\
1 \\
5 \\
28\end{array}$ & $\begin{array}{r}6 \cdot 1 \\
6 \cdot 8 \\
2 \cdot 0 \\
5 \cdot 2 \\
20 \cdot 1\end{array}$ & $\begin{array}{c}98 \\
235^{* *} \\
50 \\
96 \\
139\end{array}$ & $\begin{array}{r}43-194 \\
148-357 \\
2-236 \\
38-202 \\
99-191\end{array}$ & $\begin{array}{r}22 \\
18 \\
4 \\
23 \\
67\end{array}$ & $\begin{array}{r}17 \cdot 7 \\
22 \cdot 2 \\
4 \cdot 7 \\
16 \cdot 5 \\
61 \cdot 1\end{array}$ & $\begin{array}{r}124 \\
81 \\
85 \\
139 \\
110\end{array}$ & $\begin{array}{l}84-177 \\
52-120 \\
29-195 \\
95-197 \\
89-134\end{array}$ & $\begin{array}{r}7 \\
5 \\
1 \\
1 \\
14\end{array}$ & $\begin{array}{r}6 \cdot 6 \\
8 \cdot 2 \\
1 \cdot 8 \\
5 \cdot 9 \\
22 \cdot 5\end{array}$ & $\begin{array}{r}106 \\
61 \\
56 \\
17 \\
62\end{array}$ & $\begin{array}{c}50-199 \\
24-128 \\
2-263 \\
1-80 \\
38-97\end{array}$ \\
\hline
\end{tabular}

$=$ Confidence interval.

,$<0.01$. 
Table 6 Mortality 1900-80 from all causes by cohort of entry and by birth cohort, for men alive 15 years after entry

\begin{tabular}{|c|c|c|c|c|c|c|c|c|c|c|c|c|}
\hline \multirow[t]{2}{*}{ Years of entry } & \multicolumn{4}{|c|}{ Born 1880-99 } & \multicolumn{4}{|c|}{ Born 1900 } & \multicolumn{4}{|l|}{ Total } \\
\hline & Obs & $\operatorname{Exp}$ & $S M R$ & $90 \% \mathrm{CI}$ & Obs & $\operatorname{Exp}$ & $S M R$ & $90 \% \mathrm{CI}$ & Obs & $\operatorname{Exp}$ & $S M R$ & $90 \% C I$ \\
\hline $\begin{array}{l}1900-29 \\
1930-49 \\
1950-64 \\
\text { Total }\end{array}$ & $\begin{array}{r}134 \\
29 \\
2 \\
165\end{array}$ & $\begin{array}{r}101.5 \\
32.7 \\
0.7 \\
134.9\end{array}$ & $\begin{array}{l}132^{* * *} \\
89 \\
286 \\
122^{* *}\end{array}$ & $\begin{array}{r}114-152 \\
63-121 \\
49-898 \\
107-139\end{array}$ & $\begin{array}{r}51 \\
68 \\
14 \\
133\end{array}$ & $\begin{array}{r}54 \cdot 2 \\
78 \cdot 2 \\
16 \cdot 2 \\
148 \cdot 6\end{array}$ & $\begin{array}{l}94 \\
87 \\
85 \\
89\end{array}$ & $\begin{array}{l}74-119 \\
70-106 \\
52-135 \\
77-103\end{array}$ & $\begin{array}{r}185 \\
97 \\
16 \\
298\end{array}$ & $\begin{array}{r}155 \cdot 7 \\
110 \cdot 9 \\
17 \cdot 0 \\
283 \cdot 4\end{array}$ & $\begin{array}{c}119^{* *} \\
87 \\
94 \\
105\end{array}$ & $\begin{array}{r}105-134 \\
73-104 \\
59-143 \\
95-116\end{array}$ \\
\hline
\end{tabular}

$\mathrm{CI}=$ Confidence interval.

${ }^{* *} \mathrm{p}<0-01,{ }^{* * *} \mathrm{p}<0.001$.

Table 7 Mortality 1942-80 from all causes and from selected causes of death below age 70 among men furst employed in 1900-29 and born in 1880-99 (men alive 15 years after entry)

\begin{tabular}{lrrrr}
\hline Cause of death (ICD code, 8th rev) & Obs & Exp & $S M R$ & $90 \% C I$ \\
\hline All causes (0-999) & 65 & $41 \cdot 7$ & $156^{* * *}$ & $125-192$ \\
All neoplasms (140-239) & 19 & $12 \cdot 1$ & $157^{*}$ & $103-230$ \\
Digestive cancers (150-159) & 9 & $5 \cdot 1$ & 176 & $92-308$ \\
Respiratory cancers (161-163) & 6 & $2 \cdot 6$ & $231^{*}$ & $101-455$ \\
$\quad$ Urinary cancers (185-189) & 3 & $1 \cdot 2$ & $98-645$ \\
Circulatory disease (390-458) & 12 & $13 \cdot 3$ & $323^{* *}$ & $52-146$ \\
Violent deaths (E 800-999) & 10 & $3 \cdot 1$ & 400 & $175-547$ \\
Motor vehicle accidents(E 810-823) & 2 & $0 \cdot 5$ & $462^{* *}$ & $69-1257$ \\
Other accidents and poisonings & 6 & $1 \cdot 3$ & $180^{* *}$ & $201-911$ \\
(E 800-807, 825-949) & 6 & $13 \cdot 3$ & $412^{* *}$ & $124-254$ \\
Other causes (0-136, 240-389, & 24 & $1 \cdot 7$ & 333 & $193-773$ \\
460-796) & 7 & $0 \cdot 6$ & 235 & $58-1047$ \\
Tuberculosis (010-019) & 2 & $1 \cdot 7$ & $80-538$ \\
Peptic ulcer (531-533) & 4 & & \\
Cirrhosis of liver (571) & & & \\
\hline
\end{tabular}

$\mathrm{CI}=$ Confidence interval.

${ }^{*} \mathrm{p}<0.05,{ }^{* *} \mathrm{p}<0.01,{ }^{* * *} \mathrm{p}<0.001$.

$(\mathrm{O}=12, \mathrm{E}=11 \cdot 3, \mathrm{SMR}=106,90 \% \mathrm{CI}=61-$ 172).

\section{MORTALITY BY COHORT OF ENTRY AND BY BIRTH COHORT}

As shown in table 6, mortality from all causes was significantly raised among men first employed in the period 1900-29 and who were born before 1900 . This raised mortality is accounted for by an excess of deaths below age 70 from digestive, respiratory, and urinary cancers, motor vehicle accidents, other accidents and poisonings, tuberculosis, peptic ulcer, and cirrhosis of the liver (table 7). There is no relation between duration of exposure and mortality among this subgroup of workers, for the men alive 15 years after first employment: SMRs from all causes equal 143 for those exposed one to four years $(90 \% \mathrm{CI}=$ $103-193, \mathrm{O}=31, \mathrm{E}=21 \cdot 7), 143$ for those exposed five to 14 years $(90 \% \mathrm{CI}=86-223, \mathrm{O}=14, \mathrm{E}=$ $9 \cdot 8)$, and 127 for those still in the industry 15 years after entry $(90 \% \mathrm{CI}=106-151, \mathrm{O}=89, \mathrm{E}=70 \cdot 1)$. No significant excess mortality was observed among the men who entered the industry in the periods $1930-49$ or $1950-64$. 
contributing agent to arteriosclerosis, was never used in the compounding department. We found no evidence of management selection of less fit workers into this department but it may be relevant that smoking during work was prohibited only about a decade ago, contrary to chemical processing where it has been generally prohibited for much longer. Thus a self selection of heavy smokers to the compounding department is conceivable, although we lack any data on smoking histories. Furthermore, the numbers of deaths from lung cancer $(\mathrm{O}=2, \mathrm{E}=1 \cdot 6)$ and from chronic bronchitis $(\mathrm{O}=1, \mathrm{E}=0.3)$ are too small to be interpreted. Finally, it should be pointed out that about 50 specific causes of death are analysed for each of the four exposure categories. Therefore one can expect that an average of two significant differences at the $1 \%$ level between observed and expected deaths would arise by chance alone from the 200 comparisons. We think that chance is the more likely explanation of the excess deaths from heart disease.

Among the subgroup of workers who entered the industry before 1930 and who were born before 1900 there is an excess of deaths from a wide range of causes and there is a lack of relationship between mortality and duration of exposure. This would seem to exclude a causative occupational factor.

\section{Conclusion}

Most of the workers first employed until 1964 in the Geneva perfumery industry for at least one year have been included in the cohort, and the proportion of those not eligible for admission and of those lost to follow up was relatively low. The usual bias due to the selection of healthy workers for employment resulting in lower than expected mortality was reduced by using a 15 year latency period. This was possible because the men were traced since their entry to industry and because the follow up was long (29 years on average). The restricted size of the cohort, however, makes the comparison between observed and expected deaths from specific cancer sites and specific non-malignant causes subject to large statistical fluctuations. Consequently the present study lacks the sensitivity to detect unsuspected occupational hazards, unlike a study in which the disease is rare and specific or the mortality is greatly increased. ${ }^{67}$ We will continue the surveillance of mortality and cancer incidence among the Geneva workers but further epidemiological observation of the perfumery industry in other countries would be highly desirable.

We are indebted to Ms Patricia Dumont for handling the data of this study. We thank Mr P M Sweetnam from the MRC Epidemiology Unit, Cardiff, for help and advice during the whole study, Dr F Paccaud for critically reading the manuscript, and $\mathrm{Mr} \mathrm{I}$ D Hill for providing the MYCL program. We have benefited from the technical help of Mr A Hickson, Mr A Gross, Mr F Meier, Mr R Mermoud, Mr D Piccard, and Mrs M C Vauthier. We are grateful to Firmenich and Givaudan Companies for their collaboration.

\section{References}

' International Agency for Research on Cancer. IARC monographs on the evaluation of the carcinogenic risk of chemicals to humans. Lyon: IARC, 1982:56-7, 119-20, 126-8. (IARC monographs suppl 4.)

${ }^{2}$ Hill ID. Computing man years at risk. Br J Prev Soc Med 1972;26:132-4.

${ }^{3}$ Fox AJ, Collier PF. Low mortality rates in industrial cohort studies due to selection for work and survival in the industry. Br J Prev Soc Med 1976;30:225-30.

4 Olin GR, Ahlbom A. The cancer mortality among Swedish chemists graduated during three decades. Environ Res 1980;22: 154-61.

${ }^{5}$ Hoar SK, Pell S. A retrospective cohort study of mortality and cancer incidence among chemists. J Occup Med 1981;23:485-94.

' Fox AJ, Collier PF. Mortality experience of workers exposed to vinyl chloride monomer in the manufacture of polyvinyl chloride in Great Britain. Br J Ind Med 1977;34:1-10.

' Doll R. Relevance of epidemiology to policies for the prevention of cancer. J Occup Med 1981;23:601-09. 